\title{
Gravitational Effects on Brain and Behavior
}

\section{Laurence R. Young}

On earth, the responses of many different sensory organs normally are combined to determine our sensation of which way is down. Visual, vestibular, tactile, proprioceptive, and perhaps auditory cues are combined with knowledge of commanded voluntary movement to produce a single, usually consistent, perception of spatial orientation. Angular stabilization of the eye to reduce retinal image slip and stabilization of head and body position with respect to the vertical to avoid falling are also based upon this multisensory integration process. When tilting one's head to the shoulder, for example, this voluntary movement is confirmed to the brain by signals from the muscle and joint receptors, from the two portions of the balance mechanism of the inner ear: the semicircular canals, which sense angular motion, and the otolith organs, which sense linear acceleration and gravity. In the weightless free-fall condition of orbital space flight the correspondence among the signals is drastically altered. The otolith organs no longer indicate anything meaningful concerning the static orientation of the head. The dense mass of the otoconia no longer pulls the otolithic membrane downhill, bending the hair cell cilia of the maculae when the head is tilted. Rather, like any other linear accelerometer, the output of the otolith organs in weightlessness is limited to indications of the short-duration transient linear accelerations during head movements. Once deprived of the normal static orientation information from the otolith organs, the brain must rely upon other senses to set up a reference frame with respect to which the astronaut can judge his orientation. Visual signals play an increasing role in spatial orientation in weightlessness.

The recent Spacelab flights have provided especially valuable observations on the effects of weightlessness and space flight. During the initial states of adaptation to weightlessness, a conflict exists between the outputs of the otolith organs and the remaining senses, especially associated with voluntary head movements. This conflict is presumed to be the basis of space motion sickness, a malady which affects roughly half of all space travelers and which typically lasts two or three days. As visual cues become dominant, the astronauts begin to orient such that the surface upon which they are working becomes a vertical wall and the place where their feet touch, if they are indeed touching, becomes the floor, or the down reference. Unusual visual orientations, like seeing a fellow crew member upside down, entering a new part of the spacecraft in an unusual orientation, or looking out the window and seeing the earth at the top of the window and the sky at the bottom may prove disturbing and even bring on motion sickness symptoms. Moving visual fields create a greater sense of self motion, and otolith cues begin to be ignored as the astronaut's brain undergoes the reinterpretation of his sensory signals. The limbs no longer have any weight or require any muscle tension when static, other than what is required to overcome internal elasticity. Knowledge of limb position when the muscles are relaxed may be degraded, and the astronaut is occasionally unaware of limb position, which tends to be more flexed than in $1 \mathrm{~g}$. The ability to estimate the mass of objects, in the absence of their weight, is reduced. As measured by the Hoffman reflex during transient accelerations from weightlessness, spinal cord excitability may be greatly reduced. Ocular stabilization during head movements may be impaired, especially for the nodding and tilting motions that normally involve otolith system contributions.
In space, the otolith organs respond only to linear acceleration, as the brain may reinterpret their signals to represent only translation rather than tilt. On earth, such an interpretation would result in the wrong signals to the postural control system to prevent falling, or to reduction in the small compensatory torsional motions of the eye when tilted. Following return to earth astronauts may exhibit wide stance and unsteady gait, and difficulty balancing on a narrow rail with eyes closed. (Reduced blood supply to the brain, associated with cardiovascular deconditioning and blood pooling in the legs when standing just after reentry, may contribute to the unsteadiness.) Head movements may result in unusual illusions of self motion or of ground movement, as the otolith organ signals and perhaps the joint receptors become recalibrated to the terrestrial environment. Return to normal earth functioning may take from one or two days following short flights to several days or weeks for long flights. Part of the recovery is associated with rebuilding the leg muscles which tend to atrophy from disuse in space. Part of the readaptation is also in the brain, which must reinterpret the sensory cues appropriate for earth.

Animal experiments concerned with brain function in weightlessness have been limited. Monkeys can become motion sick, and they may show altered eye movement patterns. Goldfish, when deprived of gravito-inertial orientation forces on the graviceptors, will begin a series of looping swimming motions that may serve to satisfy a drive to remain upright. Spider webs lose their normal regularity.

The sites of adaptation to weightlessness have not yet been determined. Like many other examples of plasticity to sensorimotor rearrangement such as the wearing of reversing prisms, the adaptation is probably central. Other theories involve the end organs themselves. Space experiments can investigate changes in their morphology, such as the number and size of otoconia, or changes in the mechanics of the transducers when the steady load of $1 \mathrm{~g}$ is removed. Preliminary results concerning otolith morphology in the rat and otolith organ afferent responses in the frog are inconclusive.

See also Motion Sickness; Vestibular System; Visual-Vestibular Interactions

\section{Further reading}

Graybiel A (1973): The vestibular system. In: Bioastronautics Data Book, Parker J, West V, eds. Washington DC: National Aeronautics and Space Administration, SP-3006

Nicogossian AE, Parker JE (1982): Space Physiology and Medicine. Washington DC: National Aeronautics and Space Administration, SP-447

Special Issue on Spacelab 1 (1984): Science 225 (4658)

Young LR (1984): Perception of the body in space: Mechanisms. In: Handbook of Physiology-The Nervous System Vol 3, Sensory Processes, Part 1, Bethesda: American Physiological Society

Special Issue on Spacelab D-1 Experimental Brain Research Oct. 1986 